\title{
Developing Graduate Training Programs
}

\author{
R. H. Parsons, J. Steigler, K C Dee, G. Judd \\ Graduate School/Rensselaer Polytechnic Institute.
}

\section{Introduction}

Training Teaching Assistants (TAs) how to teach is a process that should continue throughout a student's tenure as a graduate student. In planning training programs the needs of the undergraduates as well the needs of the graduate students should be considered. The organizational framework, however, is just as important. Who will initiate programs, who will fund them? Who will run them, department, or Institute? At Rensselaer, the graduate school has struck a balance between the department and Institute. This balance allows the Graduate School to run institutional wide orientation sessions and be a consultant for departmental run TA training seminars. In this paper, we describe how the needs of the undergraduates were determined and incorperated into our orientation programs. We will also illustrate two programs: International Teaching Assistant (ITA) English class, and a mathematics teaching assistant (TA) training seminar, that have used the Graduate School for assistance in implementing their programs.

\section{What is Important to Undergraduates?}

A critical component in developing a teaching program is to determine what it is that the students need from their TAs. In order to assess the needs of our undergraduates, we surveyed students enrolled in a course titled "Introduction to Engineering Analysis" in the fall of 1993. Roughly 680 freshman engineering students (about $96 \%$ of the engineering class of 1997) had the opportunity to respond to the survey. The survey was intended to gauge undergraduate opinion about the general performance of RPI Teaching Assistants.

The survey consisted of a qualitative, short-answer section where students were asked open-ended questions about the individual "best" and "worst" TAs they interacted with. Below are the questions from the survey, followed by the overall responses from the undergraduates.

1. What qualities does your best TA have?

2. What qualities does your worst TA have?

3. Are either your best or your worst TAs international TAs?

4. What do you think are the top three things a TA should know how to do?

The following characteristics were repeatedly attributed to the "best" TAs.

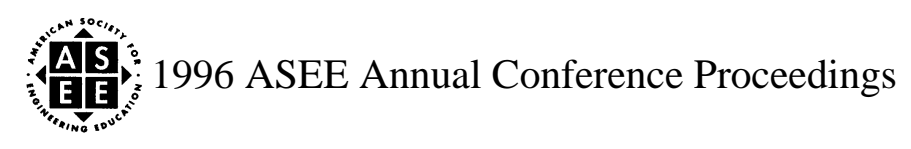


- are knowledgeable in the subject they teach

- use concise, relevant examples to clarify what is taught in class

- explain new concepts in ways that students understand

- do not assume students have prior knowledge of the subject

- show that they care about their students

- make efforts to be available for extra help

- review course material with the students before exams/finals

Some relevant quotes from the surveys:

"He writes summaries of the material covered and then e-mails them to us. His summaries explain the material very well."

"She explains what Professor ---- attempts to teach."

"He gets to know all the students well. It makes for a comfortable atmosphere where questions are easy to ask."

"She is the only reason I am passing the course."

The "worst" TAs:

- were not approachable

- were short-tempered

- ran recitations which the students considered "not helpful"

- were not prepared for class

- got "annoyed" when students asked questions

- could not explain concepts in ways that the students understood

- were not able to communicate well in English

- did not seem to understand the subject being taught

Some of the less explicit quotes on this subject:

"She openly insults me in class."

"He is ornery. Rude. Unhelpful."

"It's not their fault. There are too many students in the class to give the attention to everyone and there's not enough time."

"English. English. ENGLISH."

Finally, in response to the question "What do you think are the top three things a TA should know how to do?", nearly every response contained variations on four distinct themes:

TAs should:

- know how to teach

- know how to do the work assigned in the course 
- understand/respect students

- $\quad$ speak English

Interestingly, no complaints were received about the grading practices of individual TAs. It should be noted that complaints about TAs not understanding the subject being taught were usually paired with comments that the TA had difficulty communicating effectively (not necessarily due to problems speaking English). The TA's technical knowledge may have been more than adequate - understanding a subject and effectively teaching a subject are two entirely different matters.

From the survey responses, there were three major areas that needed to be included in the orientation: (1) English language skills, (2) communication skills, and (3). positive attitude projection

\section{TA Training Program Development}

Our approach was to address the needs of the undergraduates and new graduate TAs needs in a multi-part program consisting of orientation sessions before the academic year, peer training by other TAs, a special emphasis on English speaking capability, and an academic year seminar program.

\section{Peer TA Selection}

The orientation programs are run by current graduate TAs. These students are nominated by faculty at Rensselaer as being among the best graduate students. They are interviewed by the Graduate School Staff and a past Master TA, and selected from the nominations in what has become a very competitive process. They are given the title of Master TAs (MTA), are trained during a three day program, and compensated with an additional stipend.

\section{Summer Orientation TA Program}

Approximately 150 TAs from different departments attend our orientation programs that are run in August just prior to the beginning of Fall classes. The activities during the summer program are general to all departments, allowing the mixing of disciplines. Mixing disciplines in such a manner helps students to get to know other students outside of their department or school, and lets students benefit from the varied experiences and strengths of other disciplines. The orientation program is broken into two components, International TA Orientation, for international students only and the General TA Orientation for both international and national students.

\section{International Teaching Assistant Training}

International students have unique needs that should be met separately from other new TAs. These include cultural (particularly in the delivery of education) and language differences. These needs at Rensselaer are addressed during a two day program that is run just prior to the General Orientation Program.

There are two types of cultural differences: one is associated with the everyday activities of living (such as eating, mailing a letter, getting a checking account, etc.) and the other is associated with educational issues. These issues are dealt with in two separate sessions. The social differences are discussed in a small group session led by current international graduate students who explain their own experiences: how they learned to cope with the American way of doing things, how they have integrated their own social needs into their daily 
lives, etc. A program conducted by the International Education Office supplements the Graduate Schools efforts. The second sessions is aimed at helping the international student understand what it means to be an American educator. Generally a major difference between American and International educational practices is the level of formality and the amount of interaction between the TA and the student. This is particularly important as Rensselaer moves towards an increase in interactive learning and studio classes.

Teaching well requires communicating well. Rensselaer has instituted a program to evaluate the English verbal communication skills of the international students (all students whose first language is not English) to determine their communication proficiency. Briefly, the students are given the SPEAK test (ETS, Princeton, $\mathrm{NJ})$ which is a standardized test that requires professionally trained personnel to rank students on a scale from 0 (no communication skills) to 300 (excellent skills). From experience we have found that students below 180 cannot effectively communicate and thus, at Rensselaer, are not allowed to interact with undergraduates. They are required to take an English as a Second Language (ESL) class described below. The group of students with scores of 230 and above have good to excellent verbal skills and are allowed free interaction with undergraduates. Those students with scores between 180 - 230 can, if they have well-developed teaching skills, interact with undergraduates to varying degrees. The level of interaction for this group is decided by asking them to participate in a micro-teaching session in which they are asked to give a 15 minute presentation to the ESL Director, a Graduate School Representative, and the department graduate TA coordinator.

Incorporating a department member was found to be essential in obtaining and facilitating departmental buy-in for the resulting decisions.

\section{International Teaching Assistants English Classes}

In response to the undergraduate and ITA survey, the ESL class for ITAs changed dramatically. A cultural laboratory experience has been added to our long-standing Oral Communication for TAs classes; and American students have been actively recruited to participate in class in order to increase exposure of ITAs to other speakers of English.

These changes are supported by results of informal interviews conducted by the ESL coordinator at Rensselaer. ITAs were asked how often, outside of class, they spoke English; how much time they spent with undergraduates, what social events or clubs they joined on campus, what did they think were the obstacles they faced in learning English? The responses were shocking. Ninety eight percent of the students said they spoke no or little English outside of class. Barring an hour a week during their research group meeting, none had any informal or social contact with English-speaking undergraduates. All agreed that more contact with Americans would help their English development and understanding of American way of life. Some of the major reasons preventing them from engaging in the larger culture were:

- lack of time

- family responsibilities

- age difference between ITAs and undergraduates. "I'm married and have children. We're too different. "

- too many international students on and off campus

- isolated in married dorms or living with other international students.

- here for a degree, not culture 
The lack of interaction with English speaking individuals was addressed by including American undergraduates in the Oral Communication for ITAs course as participants in the course and as part of an outside of the classroom "Cultural Laboratory Experience".

\section{American Undergraduate Participation in ESL Classes}

In January of 1992, the first American undergraduates participated in the Oral Communication for ITAs courses. There are two classes, Oral Communication I and II, given in the Fall and Spring semester. Each class meets twice a week for an hour and twenty minutes, and has 12 to 15 students. The students in oral communication primarily come from the People's Republic of China, Taiwan, Korea, and a few from Europe, Turkey and Central and South America.

The role of the English-speaking undergraduate in Oral Communication is at times an assistant, a student participant, or speaker presenter. Since Oral Communication for TAs focuses on four main areas: speech production, teaching methodology, presentation skills, and acculturation, the undergraduate's role shifts depending on the activity. For example when the class practices pronunciation, the undergraduate models and corrects students. During group discussions, the undergraduate is a participant and at times a group facilitator. During video-tape presentations, he/she does a model presentation and then helps to tape students. Some obvious benefits are that the class can be split for correction and video-taping, giving more time for individual attention; the ITAs are exposed to different English speakers requiring them to adapt to different speaking styles; and they are in direct contact with their audience enabling them to ask questions in the context of teaching. For example when the group discussed student and TA rapport, one undergraduate said, "What is really important is for me to know that ITAs care about my progress. Knowing the subject is important, but if a TA has a negative attitude it turns me off." The ITAs listened and began discussing the important elements of establishing rapport.

An unlikely benefit to using undergraduates in Oral Communication classes arose when ITAs had to teach phonetics to the undergraduates. This gave the ITAs an opportunity to show the undergraduates how phonetic symbols work and what sounds they represent. This teaching session not only helped build the ITAs confidence as English learners but also heightened undergraduates understanding of the difficulty of learning a second language. One undergraduate commented "These( phonetic symbols) are like hieroglyphics to me. I can't remember which sound goes with what. I didn't know we had so many sounds. This is really difficult."

\section{Cultural Laboratory Experience with American Undergraduates}

The class is divided into smaller groups (6-8) for the cultural laboratory experience. The laboratories are led by an English-speaking graduate with the help of 2 to 3 English speaking undergraduates. This greatly increases the ITAs exposure to different English speakers and exposes them to their future audience of undergraduate students. The cultural laboratory is a time where ITAs, the ESL TA, and 2-3 undergraduates meet for an hour a week at the Student Union to discuss any topic(s) the group wants to talk about. Sometimes, the group watches part of a sitcom (like Seinfeld and Roseanne) and then discusses the major themes in the context of American culture and their own. Other times, a student brings in an immediate concern about landlord-tenant problems, health care, or parents' night at the elementary school. The laboratory is intended to have flexibility, and for the ITAs and undergraduates to interact in a less formal situation. The ESL TA meets with the coordinator once a week to go over planned topics and the progress of the group. In the cultural laboratory experience, the undergraduates are student participants, sharing American 
culture with the ITAs as well as learning about other cultures. Both groups benefit from this exchange: the ITA starts to know his/her audience and about American culture, whereas the undergraduates learn about many different cultures: this increases the undergraduates' acceptance and strategies for understanding English with different inflections or stresses. One undergraduate said, "I'm really glad I was in your class. I have a TA from Japan and I can understand him whereas the others (students) are lost. The first couple of weeks I was in your class, I couldn't understand anyone, but then I got to know them and got used to their accent."

Even though the cultural laboratory experience increases the required time for Oral Communication for ITAs, it has had favorable results. Student evaluations have consistently rated the laboratory highly, and worth the extra time invested. The ITAs micro-teaching results have improved with the addition of the laboratory experience.

In conclusion, incorporating the English-speaking undergraduate in the Oral communication class and adding the cultural laboratory experience has increased the interaction of the ITA and undergraduate. This exposure is the first step in developing inter-communication between the two groups. More programs like this are needed to increase understanding among the various groups on campus.

\section{General Teaching Assistant Program}

The General Orientation Program consists of three main components: Micro-teaching, a session on How to get Started, and a session focusing on common educational problems encountered by a new TA. The Microteaching is similar to that used for the International students. The new TAs are asked to prepare a 15 minute presentation and be prepared to give the talk in front of a small group (8-10) of their peers. The presentation is videotaped and the group critiques the presentation. The MTA is trained to make the session a positive experience, by for example asking the presenter to start the critiquing session. Most students are very critical of themselves and will often see mistakes they have made. This is the value of videotaping a session: the student is learning from his or her own mistakes. This allows the MTA to look for positive things that the presenter did and to point those out to the small group. This experience is a morale booster for the new graduate students as indicated on post-program surveys.

The most difficult part of the program is to help students learn how to project a positive attitude, because you are potentially asking the new TA to change their behavior. The session called "Teaching Well - How to Start" attempts to get the message across that students like to be treated with respect. The session starts with hands-on techniques for getting undergraduate students to interact with each other. There are also suggestions presented regarding various techniques for learning students' names, how to put students into groups, and how to encourage students to ask questions. However the most effective part is where the new Teaching assistants are asked the same questions we asked the undergraduates in the 1993 survey: What makes a good TA, or teacher? What makes a bad TA or teacher? Their answers are written on the blackboard or overhead projector. Not surprisingly, new Teaching Assistant come up with the same list as the undergraduates did in 1993.

The third component of the orientation covers specific issues such as: cheating, sexual harassment, re-grading an exam or homework, getting an undergraduate to academic or personal counseling, leading a discussion, running a recitation group, setting office hours, how to function in a multicultural environment, etc. Many of these issues have multiple answers to specific situations, however it is important that the new TAs know that there are answers and that they should communicate with the faculty to clearly establish their responsibilities and the faculty members policy on issues such as cheating, re-grading, etc. To stimulate discussion the new 
TAs are divided into small groups (15-20) and shown a videotape with short (1-2 min.) vignettes of these situations. The MTAs then lead a discussion on the various topics and try help the new TAs see problems that they may encounter and suggest a number of ways to handle the problems.

\section{Academic Year Program}

In addition to the summer programs, the graduate school assisted in the development of an academic year teaching seminar for new TAs. First these questions were asked: (a) What population should it address? (b) Should the seminar be discipline-based or interdisciplinary in scope? (c) Should it be optional or required? (d) Where should such an experience be housed - department, school, or across the university? (e) Who would organize and present the various topics? (f) How would if be funded? (g) How would it be evaluated? In an attempt to resolve these and other issues, it was decided to develop a seminar housed in the mathematics department with guidance and supervision from the graduate school. If this program was successful it would then be extended to other departments. The mathematics seminar would be a mandatory, one credit-hour course organized by the mathematics chair, a senior mathematics TA, and a graduate school representative. It would be taught primarily by the senior TA funded through the department (10 hour TA assignment) and the graduate school (camcorders, tripods, tapes, etc.).

The decision to have the academic year activities department based instead of interdisciplinary was important in order to gain the students attention. With all the competing activities of a new TA: new courses to take, new environment to understand, new research to begin; the new TAs needed to see the course as solving their immediate needs (how do I conduct my class tomorrow) as well as long range needs of learning how to be a good educator. The departmental seminar can focus on specific teaching strategies for that discipline and coordinate the substantive issues and disciplinary knowledge that is central to that department. For example, the mathematics TAs are interested in how to teach convergent and divergent problems, how to connect the laboratory with recitation, etc.

An experienced mathematics TA was chosen to facilitate (TA facilitator) the seminar for a number of reasons. First, research has shown that TA facilitated groups are usually more beneficial and productive for beginning TAs. Puccio (1987) has shown that TA pairing (an experienced TA with a new TA) increased mentoring and assistance in teaching. Darling (1987) observed that new TAs looked to new and experienced TAs for support and advice more than other faculty or staff members in the department. The reason cited was new TAs felt more comfortable asking for help from others in the same situation then "bothering" a faculty member. In addition, the mathematics seminar focused more on practical than theoretical training. Therefore, is was desirable to have an experience mathematics TA who knew the problems and concerns of teaching mathematics at the college level. The TA facilitator was a critical player in planning and implementing the group's ideas as well as giving feedback on the new TAs progress.

The seminar is a mandatory one-credit course that meets once a week for an hour. Although it's a required course, the major aim of the teaching seminar is to be a safe place where new TAs can speak openly and honestly about their problems and concerns without fear of retribution. In order to achieve this type of environment, these sessions are only open to new and experienced TAs . Besides giving support, the second goal of the seminar is to give the new TAs guidance and feedback on their teaching skills. In order to balance the two goals, the seminar is divided into two parts: group discussions on educational topics (such as grading, cheating, leading a problem set, etc.) and peer-teaching review. 
During group discussions, the TA facilitator introduces teaching models for the new TAs to review, discuss, and apply to their experience at Rensselaer. One excellent discussion developed when the TA facilitator brought a homework problem unmarked, and asked the TAs to grade the problem and explain why they graded as they did. It turned out be an interesting discussion on grading criteria, policy and personal preference. Sometimes a new TA came in with a difficult problem and asked the group for help. The TA facilitator and other TAs would talk through the problem and offer suggestions and alternatives. In the beginning of the semester, the new TAs didn't bring up many problems or concerns but by the middle of the semester everyone had something to contribute to the discussion.

For the peer-review portion of the seminar, every other week one or two TAs would give a five minute teaching demonstration on the topic of his/her choice. The presentation, along with any visual aids or demonstrations the student felt appropriate to enhance the presentation, would be video-taped in the classroom. The other eight seminar participants along with the TA facilitator viewed the original "live presentation" as well as the video playback viewed with the student presenter. Printed evaluation forms were used to focus the discussion on relevant aspects of the effectiveness of the presentation. After this peer review, the same person gave another five minute presentation on the same or different topic, and repeated the same process of self-and-peer review. The entire review process takes about fifty minutes. Although this process is very time consuming, the "redo" presentations made dramatic improvements in the students' presentation style. It is also hoped that the TAs will see the value of self-critique and instill the importance of periodic selfevaluation throughout their teaching career.

In addition to the seminar, the new TAs enrolled in this course have classroom visitations. Nine out of ten new mathematics TAs teach two recitation sections of Math 1 and a computer generated, laboratory. During the first half of the semester the faculty member in charge of the class and the TA facilitator sit-in on a recitation session and evaluate the TAs performance. A printed evaluation form is used to ascertain the TAs strengths and weaknesses in speech delivery, non-verbal communication, student-teacher relationship, and lecturing skills. Both the faculty member and the TA facilitator fill-out the form independently and discuss each other's findings. After the observation, the TA facilitator goes back into the same recitation the following week and for twenty minutes asks the undergraduate students for their feedback. The evaluation is oral and only two questions are asked: What is working? and What needs improvement? The TA facilitator assures the undergraduates that this is purely confidential, and that nothing will be written down, that she wants to know from them how they feel about the recitation, the TA, and the class in general. Then TA facilitator and the new TA discuss the evaluation along with suggestions on ways to improve the class. Having the faculty member, experienced TA and undergraduates contribute to the evaluation process has been very beneficial for all parties concerned. For the faculty members it has sparked debate over pedagogy. For example, when and where is group work appropriate? Should the TA pick review problems or should they ask the students which problems they are having difficulty with? How would I do it? The TA facilitator has been able to share her experience with both the faculty members and new TA establishing stronger ties between faculty and TAs. For the undergraduate, it emphasized that they were part of the process and that someone was concerned and willing to listen to their opinions. For the new TA, he/she received a comprehensive evaluation.

In December, we will look at undergraduate evaluations and see if there is any significant difference from this year's evaluations to that of previous years. We will also examine the evaluations from the new TAs about the program. At this point, all parties are pleased with the course and are planning to continue it next Fall. The Graduate school is planning to approach the other departments in Science and Engineering to see whether they would be interested in starting an academic year TA program of their own. 


\section{Bibliographic Information}

Darling, A. (1987). TA Socialization: A communication perspective. Institutional Responsibilities and Responses in Employment and Education of Teaching Assistants. (pp 91-94). Columbus, Ohio State University Press

Puccio, P. (1987). Peer Counseling and Mentoring. Institutional Responsibilities and Responses in Employment and Education of Teaching Assistants. (pp 219-223). Columbus, Ohio State University Press

\section{Biographical Information}

R. H. PARSONS, Associate Dean, Graduate School/ Associate Professor, Biology

J. STEIGLER, Director, English as a Second Language Programs

K C DEE, Graduate Teaching Assistant, Biomedical Engineering

G. JUDD, Dean of the Faculty/ Dean, Graduate School 